Archived version from NCDOCKS Institutional Repository http://libres.uncg.edu/ir/asu/

\title{
Appalachỉan
}

B O O N E, N O R T H C A R O L I N A

\section{Introductory Laboratory Visual Back And Camera Upgrade: Design And Implementation For The Rankin GoTo Astronomy Facility}

\author{
By: Mariah N. Birchard, Zachary R. Pruett, Kaitlyn L. Smith, and David J. Sitar
}

\begin{abstract}
A positive introductory astronomy lab experience is essential for recruiting new prospective majors and promoting an open attitude toward the sciences for general education students. Modern and user friendly equipment is needed in order to provide students with a more engaging and successful learning experience. Digital astronomical imaging technology has advanced enormously in the past two decades; therefore, we are in the process of upgrading and augmenting our university's astronomy facility, the Rankin GoTo Astonomy Lab.
\end{abstract}

Birchard, M. N., et al. (2016). "Introductory laboratory visual back and camera upgrade: Design and implementation for the Rankin GoTo astronomy facility." The Physics Teacher 54(4): 250-251. https://doi.org/10.1119/1.4944374. Publisher version of record available at: https://aapt.scitation.org/doi/full/10.1119/1.4944374 


\section{Introductory laboratory visual back and camera upgrade: Design and imple- mentation for the Rankin GoTo astronomy facility}

Mariah N. Birchard, Zachary R. Pruett, Kaitlyn L. Smith, and David J. Sitar, Appalachian State University, Boone, NC 28608; sitardj@appstate.edu

$\Delta$ positive introductory astronomy lab experience is essential for recruiting new prospective majors and promoting an open attitude toward the sciences for general education students. Modern and user friendly equipment is needed in order to provide students with a more engaging and successful learning experience. Digital astronomical imaging technology has advanced enormously in the past two decades; therefore, we are in the process of upgrading and augmenting our university's astronomy facility, the Rankin GoTo Astonomy Lab.

\section{Motivation - Enhancing hardware and software}

With CCD technology becoming more affordable, we purchased an SBIG STF-8300C color CCD camera with a Kodak KAF-8300 chip, to replace the extant camera, which is an SBIG STV with a Texas Instruments TC-237 chip (see Fig. 1 for chip size comparison). With the purchase of the new camera we found that the current setup on our 11-inch Celestron CPC1100 Schmidt Cassegrain Telescopes (SCT) would have to be reconfigured; otherwise, the camera would not be able to clear the mount and approximately $10^{\circ}$ along the north celestial pole would not be viewable.

Four potential setups (Fig. 2) were proposed and tested during the fall 2014 and spring 2015 semesters. The first setup entailed our new camera connected to the VISTA filter box, which was connected to the back of the scope. Our second setup tested two "piggy backed" refracting telescopes, an 80$\mathrm{mm}$ Explore Scientific apochromatic refractor with a $480-\mathrm{mm}$ focal length, and an 80-mm Lunt achromatic refractor with a 560-mm focal length, on top of the SCT. For this setup we completely redesigned the VISTA, producing VISTA2. For the final setup, we created VISTA3, which has a diffraction grating, neutral density filter, wide-field lens and zoom lens. The VISTA3 and camera are connected to the back of the SCT.

As far as software, Registax6, AutoStakkert2, Mira, MaxIm DL, CCDOps, Nebulosity, and DeepSkyStacker were all tested. Keeping affordability and user friendliness in mind, we wanted easy-to-use astro-imaging software. Nebulosity best fulfilled these requirements; it is quick to learn, with excellent

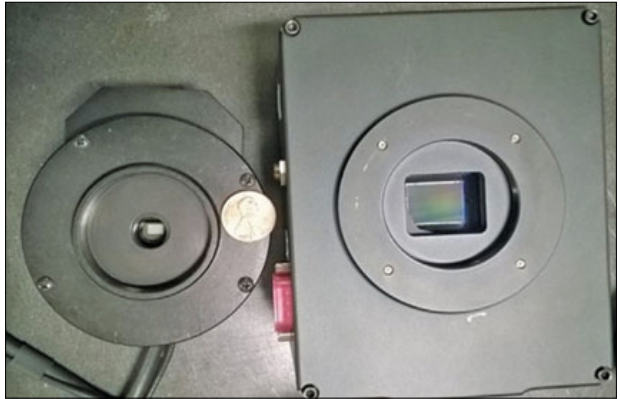

Fig. 1. Chip size comparison (STV on left and STF-8300C on right).

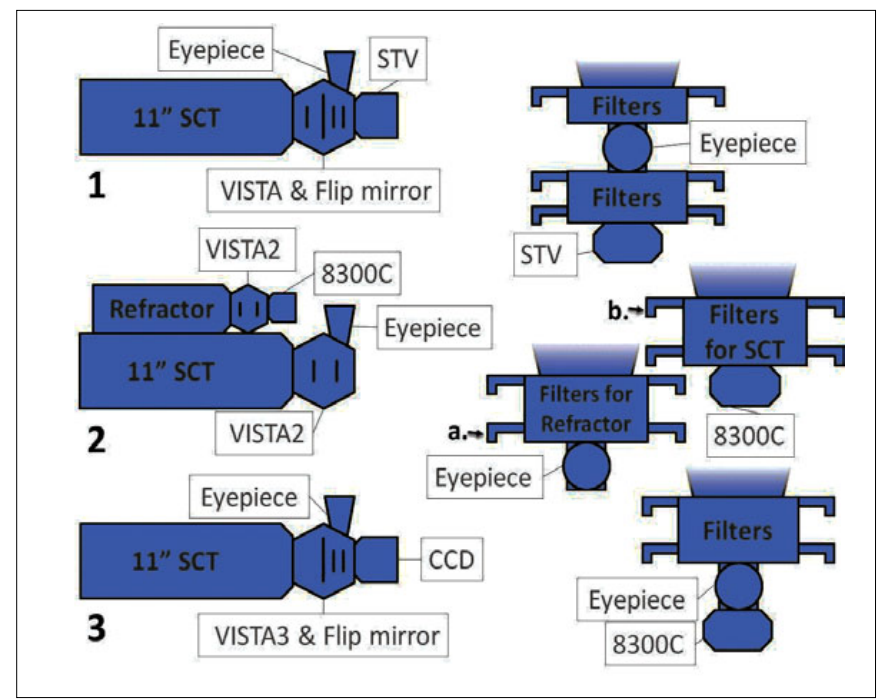

Fig. 2. (Left) test setups, (right) filter slider configurations. 1. Current older VISTA setup. 2. Refractor setups with (a) showing camera on wide-field refractor and (b) SCT with eyepiece. 3. Setup we found to most fit our needs. We hope to implement this setup into the other 14 telescopes over the summer of 2016.

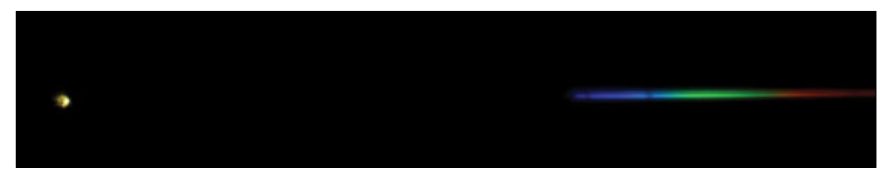

Fig. 3. Setup 3. Sirius on left with spectrum on right. Notice the dark absorption lines. With the new setup the entire visible spectrum can be seen, and the color helps students understand the relationship between color and wavelength.

tools for combining and dark subtracting images, as well as an adjustable histogram for bringing out faint detail.

RSpec, which takes a spectrum image (Fig. 3) and converts it to an interactive plot, was also tested and found to be more interactive and fun to use (Fig. 4) compared to our old method of imaging spectra (Fig. 5). Adding RSpec to our lab activities would introduce students to the real science of spectroscopy, allowing them to go beyond an image and understand some of the science behind why it looks that way. 


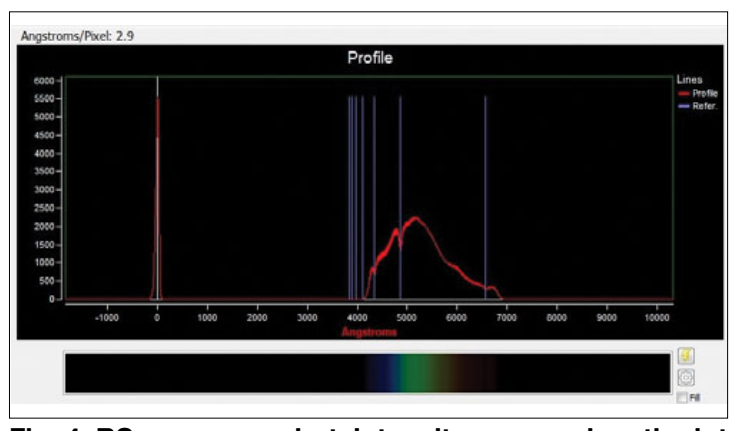

Fig. 4. RSpec screenshot, intensity-vs-wavelength plot of Sirius' spectrum (Fig. 3). RSpec takes a spectrum and with just a few minutes of work produces an interactive intensity-vs.-wavelength plot. Absorption lines can be superimposed and matched to dips in the plot as shown.

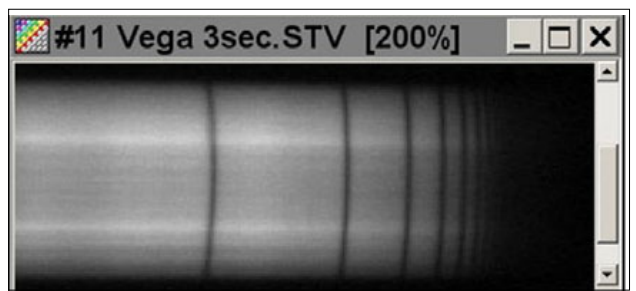

Fig. 5. Spectrum of Vega using our existing setup. The cylindrical lens in the VISTA spreads the spectrum out, making the absorption lines easier to see, but the small chip size means only a fraction of the spectrum can be seen.

\section{Results and conclusion}

The Orion Nebula was imaged for direct comparison of each setup. The extant setup's small chip size and aged technology produced the least exciting image (Fig. 6). We found that the best arrangement did not include a refractor. The wide field of view of a short focal length refractor is excellent for deep-sky astrophotography, but it did not supply the "wow!" factor we wanted to inspire in our students. The refractor produced a clear and crisp image, but did not supply great enough magnification (Fig. 7). The final setup with VISTA3 proved to be the best imaging arrangement, producing a high-resolution image with plenty of magnification (Fig. 8).

The increased chip size of the camera, when used on the SCT, provided ample room for imaging while at the same time giving impressive resolution and easily obtained, saved, and processed color images. Setup 3 paired with the image processing software Nebulosity proved to be the winning combination.

\section{Comments}

Although the refractors did not turn out to be the best choice for the new setup, they are still incredible telescopes. We will be using them in future projects to discover their complete potentials, as well as for our many astronomy outreach events in the community.

\section{Future work}

We are currently seeking funding to replace all of the old cameras and reconfigure all the VISTAs into VISTA3s. Along

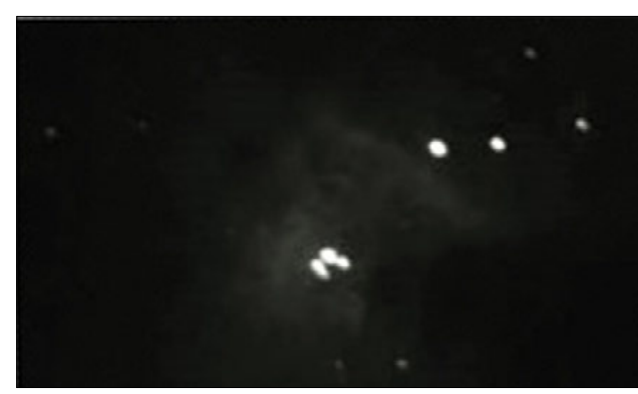

Fig. 6. Orion Nebula, existing setup (Texas Instruments Camera, VISTA, and 11-inch SCT,15-s exposure. This object cannot exceed 15-20 seconds of exposure time on this chip otherwise stars overexpose). With advances in the ease and affordability of modern imaging technology, images like this do our students a disservice.

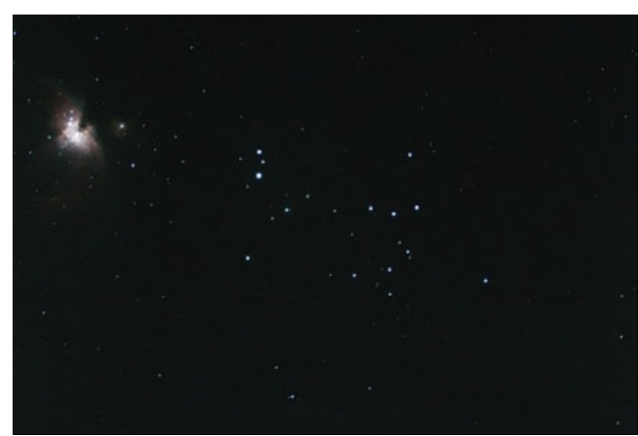

Fig. 7. Orion Nebula, Setup 2 (SBIG STF-8300C camera, VISTA2, and Explore Scientific refractor, 10 minutes of light). This lovely wide-field image was an exciting step forward, but we wanted more magnification and higher resolution.

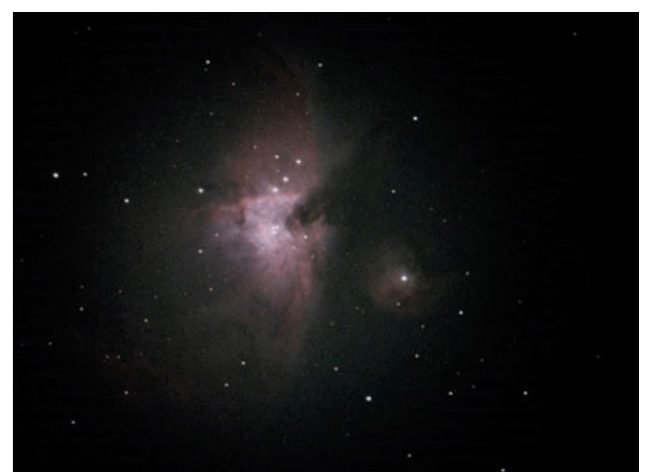

Fig. 8. Orion Nebula, Setup 3 (SBIG STF-8300C camera, VISTA3, and 11-inch SCT, eight minutes of light). Wow!

with this upgrade we would also update the imaging carts, incorporating laptops with camera control software, image processing software, and night-vision mode.

\section{Acknowledgments}

We would like to thank Lunt Engineering for loaning us the 80-mm achromatic refractor during the spring 2015 semester. In addition, we also want to thank Tom Field at RSec for letting us extend our trial period while we finished the project and Dana Greene for machining parts designed and needed. 